\title{
MORPHOMETRIC AND PHYLOGENETIC ANALYSES OF THE PALEOZOIC SUBULITOIDEA (GASTROPODA)
}

ERWIN*, Douglas, H. Dept. of Paleobiology, NHB-121, Smithsonian Institution, Washington, D.C. 20560

The gastropod superfamily Subulitoidea first appeared during the Early-Middle Ordovician gastropod radiation, seemingly from within the pleurotomarid family Lophospiridea, and persisted with low diversity and generally low abundance through the Paleozoic. One species survived the end-Permian mass extinction, but like many other Paleozoic remnants, the clade became extinct in the mid-Triassic. Many members of the clade are homeomorphic with later 'neogastropods' and have frequently been fingered as the ancestors of these later, predatory gastropods. There is however, no direct evidence for an ancestor-descendent relationship. Addressing this possibility and understanding of the systematics and evolutionary history of the clade has been complicated by relatively simple shell form and apparent paucity of shell characters. The strength of the morphological and probable ecologic similarities between subulitids and 'neogastropods' raises the question why this clade was unable to capitalize on their position as perhaps the only predatory gastropods during the Paleozoic. Such questions of evolutionary history are best addressed within the context of a combination of morphometric and phylogenetic analyses which may resolve the systematic questions and reveal something of the evolutionary relationships of the clade.

Morphometric analyses were performed on some 40 specimens covering the 13 described genera (and several undescribed forms). Both linear and angular measurements of external shell form and internal structure from axial thin sections were measured and apertures were analyzed using elliptical Fourier analysis (EFA). The number of specimens analyzed was intentionally limited because of difficulties obtaining specimens with sufficient preservation of the aperture. The results demonstrate the necessity, for this clade at least, of including a more complex description of apertural morphology than simple maximum length and width. Principle component analyses of the EFA data distinguishes complexity of the columellar folds along the first axis and basal rounding along the second - both biologically meaningful aspects of apertural form. Moreover, the occupation of a taxon-specific apertural space defined by the PCA increases through the Paleozoic. Further, the analyses suggest that the two traditionally recognized subfamilies of the Subulitidae (Ordovician-Devonian and Devonian-Triassic) each exhibit increased variance in the occupation of morphologic space, but additional data is required to confirm this pattern and determine if any temporal trends exist.

Analyzing patterns of occupation of morphologic space requires a rigorously constructed phylogeny. A cladistic (parsimony) analysis of the superfamily was performed using 16 taxa and over 30 discrete morphologic characters. The resulting cladograms were plotted within the taxon-specific morphospaces produced by the morphometric analyses. 